\title{
The clinical outcomes of second-line chemotherapy in patients with advanced pancreatic cancer: a retrospective study
}

\author{
Hyun yeb Jung, Eun Mi Lee \\ Department of Internal Medicine, Kosin University Gospel Hospital, Kosin University College of Medicine, Busan Korea
}

Background: Despite recent advances in first-line chemotherapy for advanced pancreatic cancer, standard treatment after the failure of initial chemotherapy has not been established. Hence, we aimed to retrospectively analyze the clinical characteristics and outcomes of second-line chemotherapy in patients with advanced pancreatic cancer.

Methods: We reviewed the clinical data of patients with advanced pancreatic cancer who underwent palliative chemotherapy at Kosin University Gospel Hospital between January 2013 and October 2020.

Results: Among 366 patients with advanced pancreatic cancer who had received palliative chemotherapy, 104 (28.4\%) underwent at least one cycle of second-line chemotherapy. The median age of the patients at the time of initiating second-line treatment was 62 years (interquartile range, 57-62 years), and 58.7\% (61 patients) of them were male. The common second-line chemotherapy regimens were 5-fluorouracil (FU) plus leucovorin, irinotecan, and oxaliplatin (33 patients, 31.7\%); gemcitabine/nab-paclitaxel (29, 27.9\%), gemcitabine \pm erlotinib $(13,12.5 \%)$; and oxaliplatin and 5-FU/leucovorin $(12,11.5 \%)$. The median overall survival (OS) and progression-free survival were 6.4 months (95\% confidence interval [Cl], 4.5-8.6 months) and 4.5 months (95\% $\mathrm{Cl}, 2.7-6.3$ months), respectively. In a multivariate analysis, poor performance status (PS) (hazard ratio [HR], 2.247; $p=0.021$ ), metastatic disease (HR, 2.745; $p=0.011$ ), and elevated carcinoembryonic antigen (CEA) levels $(H R, 1.939 ; p=0.030)$ at the beginning of second-line chemotherapy were associated with poor OS.

Conclusion: The survival outcome of second-line chemotherapy for advanced pancreatic cancer remains poor. However, PS, disease extent (locally advanced or metastatic), and CEA level may help determine patients who could benefit from second-line treatment.

Keywords: Chemotherapy; Pancreatic cancer; Performance status; Prognostic factor

\section{Introduction}

Although recent advances in solid tumor treatment have dramatically improved patients' survival, the prognosis of pancreatic cancer remains dismal, with a 5-year survival rate of $9 \%$ in all stages [1]. Considering that surgical resection is possible only in $15 \%-$ $20 \%$ of patients at diagnosis and most patients relapse after surgery, palliative chemotherapy is the mainstay of treatment for irresectable or recurrent diseases. Over the last decade, in two randomized phase 3 trials (the PRODIGE and MPACT trials), intensive combination chemotherapies, such as 5-fluorouracil (FU) plus leucovorin, irinotecan, and oxaliplatin (FOLFIRINOX) and gemcitabine/nab-paclitaxel, have shown a more improvement in overall survival (OS) as the initial palliative chemotherapy than gemcit-

Received: July 22, 2021 • Revised: September 7, 2021 • Accepted: September 14, 2021

Corresponding author: Eun Mi Lee, MD

Department of Internal Medicine, Kosin University Gospel Hospital, Kosin University College of Medicine, 262 Gamcheon-ro, Seo-gu, Busan 49267, Korea

Tel: +82-51-990-5820• Fax: +82-51-990-5820•E-mail: byule00@hanmail.net

Copyright (c) 2022 Yeungnam University College of Medicine, Yeungnam University Institute of Medical Science

This is an Open Access article distributed under the terms of the Creative Commons Attribution Non-Commercial License (http://creativecommons.org/licenses/by-nc/4.0/) which permits unrestricted non-commercial use, distribution, and reproduction in any medium, provided the original work is properly cited. 
abine monotherapy, which was the standard treatment until then $[2,3]$. In a phase 3 trial (NCIC CTG PA.3 trial]), the addition of erlotinib to gemcitabine demonstrated statistically significant improvement in OS compared with gemcitabine monotherapy. However, small survival gain (median OS, 6.2 months vs. 5.9 months; hazard ratio [HR], 0.82; 95\% confidence interval [CI], $0.69-0.99 ; p=0.038)$, increased toxicity risk, and high cost have limited the efficacy of the addition of erlotinib to gemcitabine [4]. Thus, FOLFRINOX and gemcitabine/nab-paclitaxel are the most widely used front-line chemotherapy regimens in patients with excellent performance status (PS), and gemcitabine monotherapy remains an option for the treatment of patients with poor OS. However, no subsequent treatment after failure of the initial chemotherapy has been established.

Three randomized phase 3 clinical trials have been conducted for second-line chemotherapy for advanced pancreatic cancer. The CONKO-003 trial showed that the combination of oxaliplatin and 5-FU/leucovorin (FOLFOX) was better in extending OS as second-line chemotherapy than 5-FU/leucovorin in patients with gemcitabine-refractory advanced pancreatic cancer (5.9 months vs. 2.3 months; HR, 0.66 ; 95\% CI, $0.48-0.91 ; p=0.010$ ) [5]. Conversely, the PANCREOX trial showed that FOLFOX did not improve OS, compared with infusional 5-FU/leucovorin (6.1 months vs. 9.9 months; HR, 1.78 ; $95 \%$ CI, $1.08-2.93 ; p=0.024)$ after gemcitabine failure [6]. This difference seems to be explained by the PS imbalance between the study groups and a possible crossover after disease progression in the PANCREOX trial. Recently, the NAPOLI-1 trial assessed the effects of nanoliposomal irinotecan, a new irinotecan formulation, alone or in combination with 5-FU/leucovorin, in patients with advanced pancreatic cancer after the failure of gemcitabine-based chemotherapy. In this trial, the group receiving nanoliposomal irinotecan combined with 5-FU/leucovorin had a longer OS than the group receiving 5-FU/ leucovorin (6.1 months vs. 4.2 months; 95\% CI, 0.49-0.92; $p=0.012)$; hence, the combination of nanoliposomal irinotecan and 5-FU/leucovorin was approved as subsequent chemotherapy after failure of gemcitabine-based chemotherapy [7].

However, these studies on second-line chemotherapy were conducted in patients who previously underwent gemcitabine, and no randomized trials focusing on treatment after the failure of a more intensive chemotherapy, such as FOLFIRINOX or gemcitabine/nab-paclitaxel have been conducted. Moreover, given that patients with advanced pancreatic cancer have different clinical characteristics and situations in a real clinical setting, their second-line treatment should be individualized. In this retrospective study, we aimed to report the clinical characteristics and results of second-line chemotherapy for patients with ad- vanced pancreatic cancer who failed initial chemotherapy in actual clinical practice.

\section{Methods}

Ethical statements: We obtained the patients' clinical features, treatment information, and outcomes from the medical records. The Institutional Review Board (IRB) of Kosin University Gospel Hospital approved this study (IRB No: KUGH 2021-07-018). The requirement for informed consent was waived because of the retrospective nature of the study.

\section{Patients}

This retrospective study reviewed the clinical data of patients with advanced pancreatic cancer who had received palliative chemotherapy at Kosin University Gospel Hospital (Busan, Korea) between January 2013 and October 2020.

We included patients who had pathologically confirmed pancreatic adenocarcinoma with locally advanced or metastatic disease and underwent at least one cycle of second-line chemotherapy. If chemotherapy was performed after the disease had progressed within 6 months of the completion of adjuvant chemotherapy, it was considered second-line chemotherapy. Histological findings other than adenocarcinoma were excluded.

\section{Statistical analysis}

Progression-free survival (PFS) was calculated from the date of starting second-line chemotherapy to the date of disease progression, and OS was calculated from the date of starting second-line chemotherapy to the date of death. The duration of clinical benefit was defined as the time interval from the time of response, including complete response (CR), partial response (PR), and stable disease (SD), to the date of disease progression. The median PFS and OS were estimated using the Kaplan-Meier method. The Cox proportional hazard model was used for univariate and multivariate analyses of prognostic factors associated with PFS and OS. Statistical analysis was performed using IBM SPSS version 23.0 (IBM Corp., Armonk, NY, USA), and statistical significance was set at $p<0.05$.

\section{Results}

\section{Patient characteristics}

This study included 366 patients diagnosed with advanced pancreatic cancer who had received palliative chemotherapy between the abovementioned periods. Among them, 104 (28.4\%) underwent 
at least one cycle of second-line chemotherapy. Table 1 summarizes the patient characteristics. The median age of the patients at the beginning of second-line treatment was 62 years (interquartile range $[\mathrm{IQR}], 57-62$ years), and $89 \%$ of the patients had an excellent Eastern Cooperative Oncology Group (ECOG) PS (0 or 1). At the time of initiating the second-line chemotherapy, 82 patients

Table 1. Baseline characteristic of patients who received second-line chemotherapy

\begin{tabular}{|c|c|}
\hline Characteristic & Data \\
\hline No. of patients & 104 \\
\hline Age $(y r)^{a)}$ & $62(57-62)$ \\
\hline Sex, male:female & 61 (58.7):43 (41.3) \\
\hline \multicolumn{2}{|l|}{ Smoking } \\
\hline Never & 74 (71.2) \\
\hline Current or former smoking & $30(28.8)$ \\
\hline Diabetes mellitus & $42(40.4)$ \\
\hline \multicolumn{2}{|l|}{ EGOG PS } \\
\hline $0-1$ & 89 (85.6) \\
\hline$\geq 2$ & $15(14.4)$ \\
\hline \multicolumn{2}{|l|}{ Primary tumor location } \\
\hline Head & $43(41.3)$ \\
\hline Body & $27(26.0)$ \\
\hline Tail & $34(32.7)$ \\
\hline \multicolumn{2}{|l|}{ Disease extent ${ }^{a)}$} \\
\hline Locally advanced & $22(21.2)$ \\
\hline Metastatic & 82 (78.8) \\
\hline \multicolumn{2}{|l|}{ Metastasis $^{\text {a) }}$} \\
\hline Liver & $53(51.0)$ \\
\hline Peritoneal & $30(28.8)$ \\
\hline Lung & $25(24.0)$ \\
\hline Bone & $9(8.7)$ \\
\hline Anemia $^{a)}$ & $87(83.7)$ \\
\hline Hypoalbuminemia) & $18(17.3)$ \\
\hline CA19-9 $(U / m L)$ & $221(41-2,166)$ \\
\hline $\mathrm{CEA}^{\mathrm{a})}(\mathrm{ng} / \mathrm{mL})$ & $6.95(3.63-25.2)$ \\
\hline \multicolumn{2}{|l|}{ Regimen of first-line chemotherapy } \\
\hline Gemcitabine \pm erlotinib & $38(36.5)$ \\
\hline Gemcitabine+nab-paclitaxel & $26(25.0)$ \\
\hline FOLFIRINOX & $34(32.7)$ \\
\hline Others ${ }^{b)}$ & $6(5.8)$ \\
\hline Response rate of first-line chemotherapy $(n=82)$ & $17(20.7)$ \\
\hline Duration of first-line chemotherapy (mo) & $4.5(2.4-7.1)$ \\
\hline
\end{tabular}

Values are presented as number only, median (interquartile range), or number (\%).

ECOG PS, Eastern Cooperative Oncology Group performance status; CA19-9, carbohydrate antigen 19-9; CEA, carcinoembryonic antigen; FOLFIRINOX, 5-fluorouracil/leucovorin, irinotecan, and oxaliplatin.

a) At start of second-line chemotherapy. ${ }^{\text {b) }}$ Gemcitabine+cisplatin, 5-fluorouracil (FU), 5-FU+leucovorin, 5-FU+cisplatin (one patient each), gemcitabine+capecitabine (two patients).
(78.8\%) had metastatic disease. As first-line chemotherapy, 38 (36.5\%), 26 (25.0\%), and 34 patients (32.7\%) received gemcitabine (with or without erlotinib), gemcitabine/nab-paclitaxel, and FOLFIRINOX, respectively. Tumor response to first-line chemotherapy was assessable in 82 patients, and response rate according to the Response Evaluation Criteria in Solid Tumor (RECIST) version 1.1 was $20.7 \%$ (none of the patients had a CR and 17 patients achieved a PR).

\section{Treatment of second-line chemotherapy}

Tables 2 and 3 list the treatment patterns and regimens used for second-line chemotherapy according to first-line regimens. For second-line chemotherapy, FOLFIRINOX, gemcitabine/nab-paclitaxel, gemcitabine \pm erlotinib, and FOLFOX were administered to $33(31.7 \%), 29(27.9 \%), 13$ (12.5\%), and 12 patients (11.5\%), respectively. Of the 38 patients who received gemcitabine \pm erlotinib as a first-line regimen, 12 (31.6\%), 8 (21.1\%), and 7 (18.4\%) received FOLFIRINOX, FOLFOX, and gemcitabine/nab-paclitaxel as their subsequent chemotherapy regimens, respectively. Of the 26 patients whose gemcitabine/nab-paclitaxel therapy failed,

Table 2. Treatment of second-line chemotherapy ( $n=104)$

\begin{tabular}{lc}
\hline Treatment & Data \\
\hline Chemotherapy regimen & $33(31.7)$ \\
FOLFIRINOX & $29(27.9)$ \\
Gemcitabine+nab-paclitaxel & $13(12.5)$ \\
Gemcitabine \pm erlotinib & $12(11.5)$ \\
FOLFOX & $7(6.7)$ \\
5-FU+cisplatin & $3(2.9)$ \\
5-FU+doxorubicin+mitomycin & $2(1.9)$ \\
Gemcitabine+cisplatin & $5(4.8)$ \\
Others & $3(2-6)$ \\
Cycle of chemotherapy & \\
Tumor response ( $\mathrm{n}=86)$ & $2(2.3)$ \\
Partial response & $33(38.4)$ \\
Stable disease & $31(36.0)$ \\
Progressive disease & $20(23.3)$ \\
Not evaluable & $4.5(2.1-7.0)$ \\
Duration of clinical benefit (mo) $(\mathrm{n}=35)$ & \\
Reason for treatment discontinuation & $52(50.0)$ \\
Disease progression & $47(45.2)$ \\
Toxicity/PS deterioration & $5(4.8)$ \\
Others & $1.9(0.6-4.6)$ \\
\hline Duration of second-line chemotherapy $(m o)$ & \\
\hline
\end{tabular}

Values are presented as number (\%) or median (interquartile range). FOLFIRINOX, 5-fluorouracil (FU)/leucovorin, irinotecan, and oxaliplatin; FOLFOX, 5-FU/leucovorin+oxaliplatin; PS, performance status.

a) 5-FU+doxorubicin, 5-FU+liposomal irinotecan+leucovorin, 5-FU, S-1, and atezolizumab (one patient each). 
Table 3. The second-line chemotherapy regimens in patients with advanced pancreatic cancer according to the first-line regimens

\begin{tabular}{|c|c|c|c|c|}
\hline \multirow{2}{*}{ The second-line regimen } & \multicolumn{4}{|c|}{ First-line regimen } \\
\hline & Gemcitabine ( \pm erlotinib) & Gemcitabine+nab-paclitaxel & FOLFIRINOX & Others \\
\hline Gemcitabine ( \pm erlotinib) & $0(0)$ & $1(3.8)$ & $12(35.3)$ & $0(0)$ \\
\hline Gemcitabine+nab-paclitaxel & $7(18.4)$ & $0(0)$ & $21(61.8)$ & $0(0)$ \\
\hline FOLFIRINOX & $12(31.6)$ & $17(65.4)$ & $0(0)$ & $4(66.7)$ \\
\hline FOLFOX & $8(21.1)$ & $3(11.5)$ & $0(0)$ & $1(16.7)$ \\
\hline 5-FU+cisplatin & $6(15.8)$ & $1(3.8)$ & $0(0)$ & $0(0)$ \\
\hline Others & $5(13.2)^{a)}$ & $4(15.4)^{b)}$ & $1(2.9)$ & $1(16.7)$ \\
\hline Total & $38(100)$ & $26(100)$ & $34(100)$ & $6(100)$ \\
\hline
\end{tabular}

Values are presented as number (\%).

FOLFIRINOX, 5-fluorouracil (FU)+leucovorin, irinotecan, and oxaliplatin; FOLFOX, 5-FU/leucovorin+oxaliplatin.

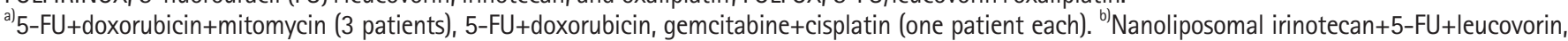
5-FU+leucovorin, gemcitabine+cisplatin, S-1 (one patient each).

20 (76.9\%) received oxaliplatin-based chemotherapy as a second-line regimen (FOLFOX and FOLFIRINOX in 3 and $17 \mathrm{pa}-$ tients, respectively). The majority of the 34 patients (97.1\%) who underwent first-line FOLFIRINOX received gemcitabine-based chemotherapy (gemcitabine \pm erlotinib and gemcitabine/nab-paclitaxel in 12 and 21 patients, respectively).

A median of three cycles of second-line chemotherapy (IQR, 2-6 cycles) was administered. Eighty-six of the 104 patients had a measurable disease based on the RECIST version 1.1, and tumor responses were assessed in these patients. None of the patients achieved a CR. Two patients had PR, and 33 patients had SD. The clinical benefit rate, including CR, PR, and SD, was 40.7\% (35 patients), and the median duration of clinical benefit was 4.5 months (IQR, 2.1-7.0 months). Among the 17 patients who had a PR in first-line chemotherapy, none achieved a PR, but 9 patients (52.9\%) attained an SD. Second-line chemotherapy was discontinued in 21 (20.2\%) patients before the first planned follow-up point due to disease progression or PS deterioration. At the first response evaluation, disease progression was observed in 31 patients (29.8\%). Furthermore, 47 patients (45.2\%) discontinued treatment because of chemotherapy toxicity or PS deterioration. Two patients died of septic shock due to chemotherapy-induced neutropenia; one had been administered gemcitabine/nab-paclitaxel and the other had been administered FOLFIRINOX.

\section{Survivals and prognostic factors}

For a median follow-up of 16.8 months, the median PFS was 4.5 months (95\% CI, 2.7-6.3 months) (Fig. 1A). Additionally, the median OS was 6.4 months (95\% CI, 4.5-8.6 months), and the 1-year survival rate was $25.3 \%$ (Fig. 1B).

Tables 4 and 5 present the univariate and multivariate analyses of potential prognostic factors associated with survival in patients with advanced pancreatic cancer who underwent second-line che-
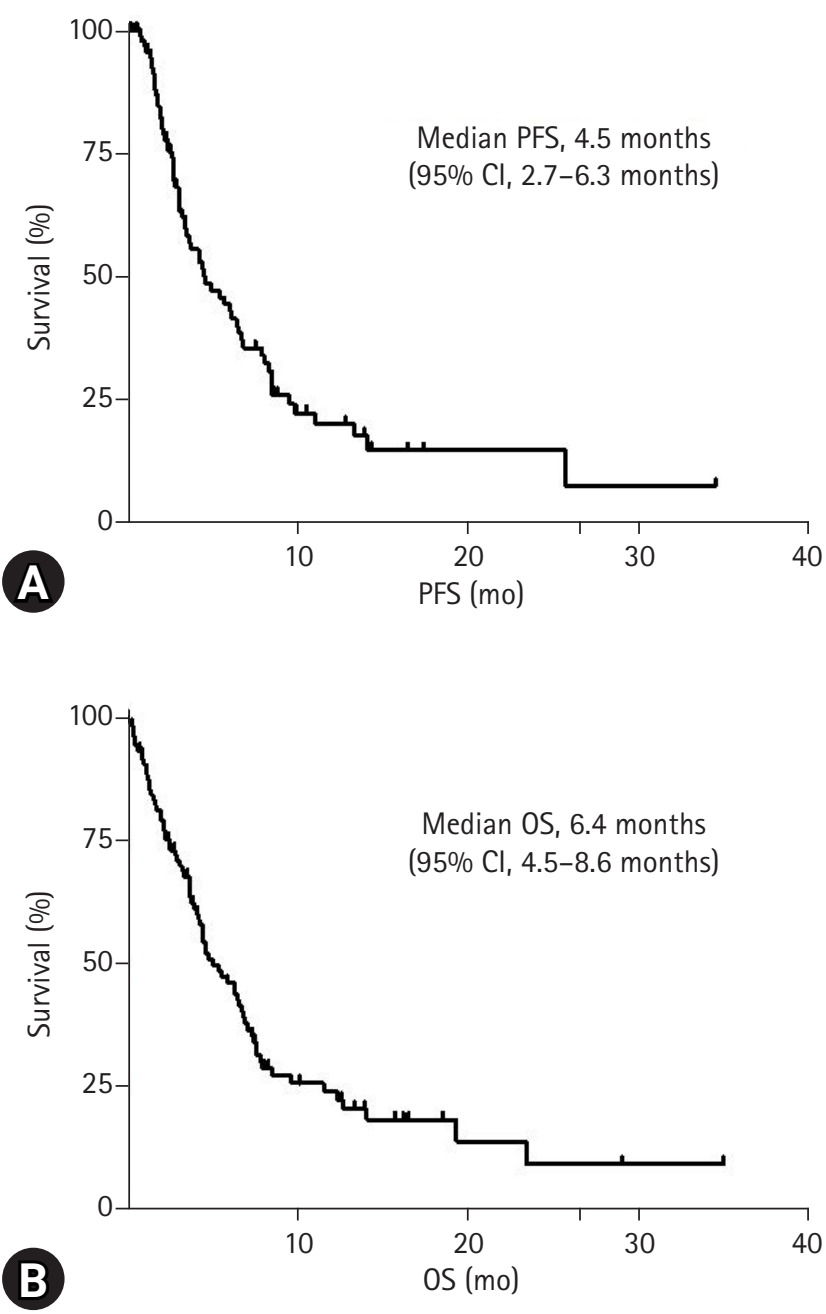

Fig. 1. Kaplan-Meier survival after second-line chemotherapy initiation. (A) Progression-free survival (PFS) after second-line chemotherapy initiation in patients with advanced pancreatic cancer. (B) Overall survival (OS) after second-line chemotherapy initiation in patients with advanced pancreatic cancer. $\mathrm{Cl}$, confidence interval. 
Table 4. Univariate and multivariate analysis on PFS for second-line chemotherapy

\begin{tabular}{|c|c|c|c|c|c|}
\hline \multirow{2}{*}{ Variable } & \multirow{2}{*}{ Median PFS (mo) } & \multicolumn{2}{|c|}{ Univariate analysis } & \multicolumn{2}{|c|}{ Multivariate analysis } \\
\hline & & $\mathrm{HR}(95 \% \mathrm{Cl})$ & $p$-value & $\mathrm{HR}(95 \% \mathrm{Cl})$ & $p$-value \\
\hline \multicolumn{6}{|l|}{ EG0G PS } \\
\hline $0-1$ & 4.5 & $1.070(0.426-2.684)$ & 0.886 & & \\
\hline$\geq 2$ & 6.0 & & & & \\
\hline \multicolumn{6}{|l|}{ Diabetes mellitus } \\
\hline No & 3.4 & $0.577(0.345-0.965)$ & 0.033 & $0.608(0.355-1.043)$ & 0.071 \\
\hline Yes & 6.8 & & & & \\
\hline \multicolumn{6}{|l|}{ Disease extent ${ }^{a)}$} \\
\hline Locally advanced & 9.8 & $3.082(1.588-5.984)$ & 0.001 & $2.728(1.205-6.178)$ & $0.016^{*}$ \\
\hline Metastatic & 3.6 & & & & \\
\hline \multicolumn{6}{|l|}{ Liver metastasis ${ }^{\text {a) }}$} \\
\hline No & 6.1 & $1.811(1.103-2.976)$ & 0.017 & $1.080(0.580-2.011)$ & 0.808 \\
\hline Yes & 3.2 & & & & \\
\hline \multicolumn{6}{|l|}{ Lung metastasis ${ }^{\mathrm{a}}$} \\
\hline No & 4.9 & $1.797(1.005-3.215)$ & 0.045 & $1.158(0.606-2.211)$ & 0.657 \\
\hline Yes & 3.4 & & & & \\
\hline \multicolumn{6}{|l|}{ Bone metastasis ${ }^{\mathrm{a}}$} \\
\hline No & 5.4 & $5.512(2.094-12.677)$ & $<0.001$ & $3.143(1.150-8.592)$ & $0.026^{*}$ \\
\hline Yes & 1.8 & & & & \\
\hline \multicolumn{6}{|l|}{ CA19-9 (U/mL) } \\
\hline$<221$ & 5.7 & $1.016(0.618-1.670)$ & 0.951 & & \\
\hline$\geq 221$ & 3.7 & & & & \\
\hline \multicolumn{6}{|l|}{$\mathrm{CEA}^{\mathrm{a})}(\mathrm{ng} / \mathrm{mL})$} \\
\hline Normal $(\leq 5.5)$ & 5.7 & $1.197(0.705-2.033)$ & 0.507 & & \\
\hline Elevation (> 5.5) & 4.2 & & & & \\
\hline
\end{tabular}

PFS, progression-free survival; HR, hazard ratio; $\mathrm{Cl}$, confident interval; ECOG PS, Eastern Cooperative Oncology Group performance status; CA19-9, carbohydrate antigen 19-9; CEA, carcinoembryonic antigen.

${ }^{a)}$ At start of second-line chemotherapy.

${ }^{*} p<0.05$.

motherapy. Univariate analysis revealed that the absence of diabetes mellitus, the presence of metastatic disease, liver metastasis, and bone metastases at the time of initiating second-line treatment were associated with lower PFS. In the multivariate analysis, metastatic disease (HR, 2.728; 95\% CI, 1.205-6.178; $p=0.016)$ and bone metastasis (HR, 3.143; 95\% CI, 1.150-8.592; $p=0.026$ ) at the time of initiating second-line were statistically significant (Table 4). In the univariate analysis of OS, poor ECOS PS ( $\geq 2)$, metastatic disease, liver metastasis, bone metastasis, and elevated serum carcinoembryonic antigen (CEA) levels at the time of initiating the second-line treatment were associated with lower OS. Among these factors, poor ECOG PS ( $\geq 2$ ) (HR, 2.247; 95\% CI, 1.129-4.474; $p=0.021)$, metastatic disease (HR, 2.745; 95\% CI, $1.260-5.983 ; p=0.011$ ), and elevated serum CEA level (HR, $1.939 ; 95 \% \mathrm{CI}, 1.065-3.530 ; p=0.030)$ at the time of initiating the second-line treatment were statistically significant in the multivariate analysis (Table 5).

\section{Discussion}

Recently, intensive initial chemotherapy using FOLFIRINOX or gemcitabine/nab-paclitaxel has improved the survival of patients with advanced pancreatic cancer [2,3]. However, the prognosis of advanced pancreatic cancer remains poor, with no standard treatment after the failure of initial chemotherapy. Hence, the present study retrospectively analyzed the clinical characteristics and outcomes of subsequent chemotherapy in patients with advanced pancreatic cancer after the failure of initial chemotherapy in clinical practice. The median OS after the start of second-line chemotherapy was 6.4 months (95\% CI, 4.5-8.6 months), similar to the recent retrospective reports of second-line chemotherapy for advanced pancreatic cancer in the real-world setting (5.2-8.1 months) [8$10]$. Excellent PS, locally advanced disease, and normal CEA level at the time of second-line treatment initiation were associated with better OS. 
Table 5. Univariate and multivariate analysis on OS for second-line chemotherapy

\begin{tabular}{|c|c|c|c|c|c|}
\hline \multirow{2}{*}{ Variable } & \multirow{2}{*}{ Median OS (mo) } & \multicolumn{2}{|c|}{ Univariate analysis } & \multicolumn{2}{|c|}{ Multivariate analysis } \\
\hline & & $\mathrm{HR}(95 \% \mathrm{Cl})$ & $p$-value & $\mathrm{HR}(95 \% \mathrm{Cl})$ & $p$-value \\
\hline \multicolumn{6}{|l|}{$\mathrm{EGOG} \mathrm{PS}^{\mathrm{a})}$} \\
\hline $0-1$ & 7.2 & $2.367(1.330-5.217)$ & 0.004 & $2.247(1.129-4.474)$ & $0.021^{*}$ \\
\hline$\geq 2$ & 2.3 & & & & \\
\hline \multicolumn{6}{|l|}{ Diabetes mellitus } \\
\hline No & 5.9 & $0.784(0.488-1.258)$ & 0.311 & & \\
\hline Yes & 8.3 & & & & \\
\hline \multicolumn{6}{|l|}{ Disease extent ${ }^{a)}$} \\
\hline Locally advanced & 29.4 & 3.380 (1.719-6.644) & $<0.001$ & $2.745(1.260-5.983)$ & $0.011^{*}$ \\
\hline Metastatic & 5.5 & & & & \\
\hline \multicolumn{6}{|l|}{ Liver metastasis ${ }^{a)}$} \\
\hline No & 8.0 & 2.780 (1.678-4.607) & $<0.001$ & $1.324(0.714-2.456)$ & 0.373 \\
\hline Yes & 5.5 & & & & \\
\hline \multicolumn{6}{|l|}{ Lung metastasis ${ }^{\text {a) }}$} \\
\hline No & 8.0 & $1.340(0.793-2.264)$ & 0.272 & & \\
\hline Yes & 4.8 & & & & \\
\hline \multicolumn{6}{|l|}{ Bone metastasis $^{\text {a) }}$} \\
\hline No & 8.0 & $2.596(1.215-5.547)$ & 0.011 & $2.136(0.964-4.737)$ & 0.062 \\
\hline Yes & 4.9 & & & & \\
\hline \multicolumn{6}{|l|}{ CA19-9 (U/mL) } \\
\hline$<221$ & 8.0 & $1.396(0.875-2.226)$ & 0.16 & & \\
\hline$\geq 221$ & 5.7 & & & & \\
\hline \multicolumn{6}{|l|}{$\mathrm{CEA}^{\mathrm{a})}(\mathrm{ng} / \mathrm{mL})$} \\
\hline Normal $(\leq 5.5)$ & 9.3 & $2.006(1.177-6.419)$ & 0.009 & 1.939 (1.065-3.530) & $0.030^{*}$ \\
\hline Elevation (> 5.5) & 5.4 & & & & \\
\hline
\end{tabular}

OS, overall survival; $\mathrm{HR}$, hazard ratio; $\mathrm{Cl}$, confident interval; ECOG PS, Eastern Cooperative Oncology Group performance status; CA19-9, carbohydrate antigen 19-9; CEA, carcinoembryonic antigen.

${ }^{\text {a) } A t ~ s t a r t ~ o f ~ s e c o n d-l i n e ~ c h e m o t h e r a p y . ~}$

${ }^{*} p<0.05$.

Advanced pancreatic cancer progresses quickly, and once it begins to progress after the initial treatment, the patient's PS deteriorates rapidly; hence, the decision to perform subsequent treatment is challenging. Nagrial et al. [11] systematically reviewed 24 prospective clinical trials of subsequent chemotherapy in patients with advanced pancreatic cancer who previously received gemcitabine-based chemotherapy between 1988 and 2012. They reported that $43 \%$ of the patients underwent subsequent chemotherapy and that the utilization rate significantly increased from studies published pre-2007 to those published post-2007 (35\%-48\%; $p=0.0015)$. The MPACT and PRODIGE trials reported that the rates of utilizing second-line chemotherapy were $38 \%$ and $47 \%$, respectively [2,3]. In our study, it was $28.4 \%$, which was slightly lower than in these prospective studies, possibly because the prospective clinical trials generally included patients with a better PS than in actual clinical practice.

After the failure of initial chemotherapy in patients with ad- vanced pancreatic cancer, those who only received supportive care showed dismal survival (approximately 2 months) compared with those who underwent second-line treatment [12]. The CONKO phase 3 trial demonstrated that OS was longer when administering FOLFOX as a second-line treatment than providing the best supportive care in patients with advanced pancreatic cancer $(4.8$ months vs. 2.3 months; $95 \%$ CI, $0.24-0.83$ months; $p=0.008)$; however, this trial was stopped prematurely because of insufficient recruitment (best supportive care was not accepted by patients and physicians) [13]. Although the clinical benefit of second-line chemotherapy is marginal, survival can be improved by providing appropriate subsequent treatment in selected patients. Therefore, patients who will benefit from subsequent chemotherapy after failure of the initial treatment must be identified.

Several studies have reported factors that can predict the survival outcomes of patients who undergo second-line chemotherapy [9,10,14-18]. Among several clinical and biochemical variables, PS 
is one of the most common and important prognostic factors in patients receiving second-line chemotherapy [9,14-17]. Consistent with these studies, patients with an excellent ECOG PS score (0 or 1 ) in our study had longer survival than those with poor PS. Patients with an excellent PS could be offered aggressive treatment to prolong their survival and manage their symptoms. Therefore, PS should be regarded as an important prognostic factor in patients receiving not only second-line but also first-line chemotherapy. A meta-analysis of 12 phase 3 randomized studies for the firstline chemotherapy of metastatic pancreatic cancer demonstrated that patients with a poor ECOG PS had a worse prognosis than those with an excellent ECOG PS (HR for OS, 1.45; 95\% CI, $1.21-1.74 ; p<0.001)$ [19]. PS was also considered in selecting the initial chemotherapy regimen. Intensive chemotherapy regimens, such as FOLFIRINOX or gemcitabine/nab-paclitaxel, are recommended for patients with an excellent ECOG PS (0 or 1$)$, whereas gemcitabine monotherapy is an option for the treatment of patients with an ECOG PS 2 [20,21].

Apart from excellent PS, locally advanced disease and normal CEA level at the time of initiating the second-line chemotherapy were associated with prolonged OS in our study. Our study revealed that locally advanced disease was associated with better survival, which is consistent with other retrospective studies $[9,17]$. In several studies, serum CA 19-9 levels at the beginning of secondline chemotherapy were reported as a prognostic factor, but in our study, this factor showed no significance $[10,15,17]$. Meanwhile, CEA level was associated with OS in our study, but other studies showed no such association; thus, it remains unclear whether CEA level is a significant prognostic factor. Considering the heterogeneity of patients who underwent second-line treatment, the prognostic factors of survival outcomes have been reported differently and, have not been established. Vienot et al. [16] analyzed a large cohort of 395 patients with advanced pancreatic cancer and developed a prognostic nomogram to predict patient survival for second-line chemotherapy. They identified nine independent prognostic factors: age, smoking status, liver metastasis, PS, pain, jaundice, ascites, duration of first-line chemotherapy, and second-line chemotherapy type. Given the lack of unified prognostic factors to predict patient survival for second-line chemotherapy, further prospective clinical studies are needed to validate these variables.

Randomized trials on subsequent chemotherapy for patients with advanced pancreatic cancer who failed in the initial chemotherapy are limited, and no acceptable standard regimen for subsequent chemotherapy has been established. The only second-line chemotherapy regimen that showed a survival benefit in a phase 3 trial is the combination of nanoliposomal irinotecan and 5-FU/ leucovorin (the NAPOLRI trial) [7]. However, the NAPOLRI tri- al did not include patients who underwent first-line FOLFIRINOX, and the value of using such combined regimen after FOLFIRINOX remains vague. In our study, only one patient received a second-line regimen containing nanoliposomal irinotecan, probably because nanoliposomal irinotecan is not covered by public insurance in Korea.

The optimal sequence of palliative chemotherapy for advanced pancreatic cancer remains unclear. Generally, first-line chemotherapy regimens and PS are considered when selecting second-line regimens. For patients who were administered prior gemcitabine-based regimens, 5-FU-based regimens are acceptable subsequent treatment options. Gemcitabine-based regimens can be administered to patients previously treated with 5-FU-based chemotherapy. Intensive chemotherapy regimens, such as FOLFIRINOX and gemcitabine/nab-paclitaxel, can be considered after failure of gemcitabine-based regimens and FOLFIRINOX, respectively, but no randomized trials have been conducted. In a single-arm phase 2 trial, administering FOLFIRINOX after gemcitabine failure showed a promising outcome, with a median OS of 8.5 months (95\% CI, 5.6-11.4 months), but $41.0 \%$ of the patients developed grade 3 or 4 neutropenia despite using an attenuated regimen [22].

Administering gemcitabine/nab-paclitaxel after first-line FOLFIRINOX failure showed a better median OS of 8.8 months (95\% CI, 6.2-9.7 months) in the AGEO trial than gemcitabine monotherapy (3.6-5.7 months) in several retrospective trials [23-26]. However, grade 3 or 4 adverse events occurred in $40 \%$ of the patients [23]. Second-line FOLFIRINOX and gemcitabine/ nab-paclitaxel have shown promising OS, but with high toxicities; thus, they should be administered to patients with an excellent PS and a favorable comorbidity profile. In our study, FOLFIRINOX was administered to $45.3 \%$ of the patients who previously underwent gemcitabine-based chemotherapy ( 29 of 64 patients), and gemcitabine/nab-paclitaxel was administered to $61.8 \%$ of the patients who failed FOLFIRINOX ( 21 of 34 patients).

This study has several limitations. First, it has a retrospective design; thus, all data were only acquired by reviewing the medical records. Therefore, the results of prognostic factors to predict survival outcomes should be interpreted with caution. Second, the patients received various chemotherapy regimens; hence, defining the benefit of a certain regimen for second-line chemotherapy is inappropriate. Further clinical studies are needed to determine the appropriate sequences for chemotherapy. In addition, the characteristics of the patients included in this retrospective analysis were heterogeneous, and the duration of second-line chemotherapy was short (median, 1.9 months). Because of these limitations, our analysis is insufficient to verify the actual effects of second-line chemo- 
therapy for advanced pancreatic cancer, and caution is needed to interpret these outcomes.

In conclusion, our findings revealed that the clinical outcome of second-line chemotherapy for advanced pancreatic cancer is still poor, with a median OS of 6.4 months (95\% CI, 4.5-8.6 months), which is consistent with other retrospective studies. Nonetheless, some factors such as PS, disease extent (locally advanced or metastatic), and CEA level at the beginning of second-line treatment could help identify patients who may benefit from second-line chemotherapy. However, because this was a small retrospective study including patients with heterogeneous characteristics, the results of this analysis should be cautiously interpreted, and further prospective clinical trials are needed to evaluate the effect of second-line chemotherapy on advanced pancreatic cancer.

\section{Notes}

\section{Conflicts of interest}

No potential conflict of interest relevant to this article was reported.

\section{Funding}

None.

\section{Author contributions}

Conceptualization, Data curation, EML; Writing-original draft: EML, HyJ; Writing-review \& editing: EML, HyJ.

\section{ORCID}

Hyun yeb Jung, https://orcid.org/0000-0001-7900-6277

Eun Mi Lee, https://orcid.org/0000-0002-0746-8883

\section{References}

1. Siegel RL, Miller KD, Jemal A. Cancer statistics, 2020. CA Cancer J Clin 2020;70:7-30.

2. Conroy T, Desseigne F, Ychou M, Bouché O, Guimbaud R, Bécouarn Y, et al. FOLFIRINOX versus gemcitabine for metastatic pancreatic cancer. N Engl J Med 2011;364:1817-25.

3. Von Hoff DD, Ervin T, Arena FP, Chiorean EG, Infante J, Moore M, et al. Increased survival in pancreatic cancer with nab-paclitaxel plus gemcitabine. N Engl J Med 2013;369:1691703.

4. Moore MJ, Goldstein D, Hamm J, Figer A, Hecht JR, Gallinger $\mathrm{S}$, et al. Erlotinib plus gemcitabine compared with gemcitabine alone in patients with advanced pancreatic cancer: a phase III trial of the National Cancer Institute of Canada Clinical Trials
Group.J Clin Oncol 2007;25:1960-6.

5. Oettle H, Riess H, Stieler JM, Heil G, Schwaner I, Seraphin J, et al. Second-line oxaliplatin, folinic acid, and fluorouracil versus folinic acid and fluorouracil alone for gemcitabine-refractory pancreatic cancer: outcomes from the CONKO-003 trial.J Clin Oncol 2014;32:2423-9.

6. Gill S, Ko YJ, Cripps C, Beaudoin A, Dhesy-Thind S, Zulfiqar $\mathrm{M}$, et al. PANCREOX: a randomized phase III study of fluorouracil/leucovorin with or without oxaliplatin for second-line advanced pancreatic cancer in patients who have received gemcitabine-based chemotherapy. J Clin Oncol 2016;34:3914-20.

7. Wang-Gillam A, Li CP, Bodoky G, Dean A, Shan YS, Jameson G, et al. Nanoliposomal irinotecan with fluorouracil and folinic acid in metastatic pancreatic cancer after previous gemcitabine-based therapy (NAPOLI-1): a global, randomised, open-label, phase 3 trial. Lancet 2016;387:545-57.

8. Ramaswamy A, Parthiban S, Malhotra M, Kothari R, Goel A, Bhargava $\mathrm{P}$, et al. Outcomes with second-line chemotherapy in advanced pancreatic cancers: a retrospective study from a tertiary cancer center in India. Indian J Cancer 2018;55:144-7.

9. Tsang ES, Wong HL, Wang Y, Renouf DJ, Cheung WY, Lim HJ, et al. Outcomes and characteristics of patients receiving second-line therapy for advanced pancreatic cancer. Am J Clin Oncol 2019;42:196-201.

10. Gränsmark E, Bågenholm Bylin N, Blomstrand H, Fredrikson M, Åvall-Lundqvist E, Elander NO. Real world evidence on second-line palliative chemotherapy in advanced pancreatic cancer. Front Oncol 2020;10:1176.

11. Nagrial AM, Chin VT, Sjoquist KM, Pajic M, Horvath LG, Biankin AV, et al. Second-line treatment in inoperable pancreatic adenocarcinoma: a systematic review and synthesis of all clinical trials. Crit Rev Oncol Hematol 2015;96:483-97.

12. Rahma OE, Duffy A, Liewehr DJ, Steinberg SM, Greten TF. Second-line treatment in advanced pancreatic cancer: a comprehensive analysis of published clinical trials. Ann Oncol 2013;24:1972-9.

13. Pelzer U, Schwaner I, Stieler J, Adler M, Seraphin J, Dörken B, et al. Best supportive care (BSC) versus oxaliplatin, folinic acid and 5-fluorouracil (OFF) plus BSC in patients for second-line advanced pancreatic cancer: a phase III-study from the German CONKO-study group. Eur J Cancer 2011;47:1676-81.

14. Kim ST, Choi YJ, Park KH, Oh SC, Seo JH, Shin SW, et al. A prognostic model to identify patients with advanced pancreas adenocarcinoma who could benefit from second-line chemotherapy. Clin Oncol (R Coll Radiol) 2012;24:105-11.

15. Sinn M, Dälken L, Striefler JK, Bischoff S, Schweitzer N, Pelzer $\mathrm{U}$, et al. Second-Line treatment in pancreatic cancer patients: 
who profits? Results from the CONKO Study Group. Pancreas 2016;45:601-5.

16. Vienot A, Beinse G, Louvet C, de Mestier L, Meurisse A, Fein F, et al. Overall survival prediction and usefulness of second-line chemotherapy in advanced pancreatic adenocarcinoma. J Natl Cancer Inst 2017;109. doi: 10.1093/jnci/djx037.

17. Hsu CC, Liu KH, Chang PH, Chen PT, Hung CY, Hsueh SW, et al. Development and validation of a prognostic nomogram to predict survival in patients with advanced pancreatic cancer receiving second-line palliative chemotherapy. J Gastroenterol Hepatol 2020;35:1694-703.

18. Lee JE, Lee HS, Chung MJ, Park JY, Park SW, Song SY, et al. Analysis of clinical predictive factors affecting the outcome of second-line chemotherapy for gemcitabine-refractory advanced pancreatic cancer. Gut Liver 2020;14:135-43.

19. Colloca G. Performance status as prognostic factor in phase III trials of first-line chemotherapy of unresectable or metastatic pancreatic cancer: a trial-level meta-analysis. Asia Pac J Clin Oncol 2021 Jun 23 [Epub]. http://doi.org/10.1111/ajco.13598.

20. Sohal D, Kennedy EB, Khorana A, Copur MS, Crane CH, Garrido-Laguna I, et al. Metastatic pancreatic cancer: ASCO clinical practice guideline update. J Clin Oncol 2018;36:2545-56.

21. Ducreux M, Cuhna AS, Caramella C, Hollebecque A, Burtin P, Goéré D, et al. Cancer of the pancreas: ESMO Clinical Practice
Guidelines for diagnosis, treatment and follow-up. Ann Oncol 2015;26(Suppl 5):v56-68.

22. Kim JH, Lee SC, Oh SY, Song SY, Lee N, Nam EM, et al. Attenuated FOLFIRINOX in the salvage treatment of gemcitabine-refractory advanced pancreatic cancer: a phase II study. Cancer Commun (Lond) 2018;38:32.

23. Portal A, Pernot S, Tougeron D, Arbaud C, Bidault AT, de la Fouchardière $\mathrm{C}$, et al. Nab-paclitaxel plus gemcitabine for metastatic pancreatic adenocarcinoma after FOLFIRINOX failure: an AGEO prospective multicentre cohort. Br J Cancer 2015; 113:989-95.

24. da Rocha Lino A, Abrahão CM, Brandão RM, Gomes JR, Ferrian AM, Machado MC, et al. Role of gemcitabine as second-line therapy after progression on FOLFIRINOX in advanced pancreatic cancer: a retrospective analysis. J Gastrointest Oncol 2015;6:511-5

25. Viaud J, Brac C, Artru P, Le Pabic E, Leconte B, Bodère A, et al. Gemcitabine as second-line chemotherapy after FOLFIRINOX failure in advanced pancreatic adenocarcinoma: a retrospective study. Dig Liver Dis 2017;49:692-6.

26. Sarabi M, Mais L, Oussaid N, Desseigne F, Guibert P, De La Fouchardiere C. Use of gemcitabine as a second-line treatment following chemotherapy with FOLFIRINOX for metastatic pancreatic adenocarcinoma. Oncol Lett 2017;13:4917-24. 